\title{
New Tumor Event After Initial Treatment Present
}

National Cancer Institute

\section{Source}

National Cancer Institute. New Tumor Event After Initial Treatment Present. NCI

Thesaurus. Code C156850.

There is evidence of a new tumor after the initial treatment. 